\title{
What's the Catch? Reducing Consumption of Contaminated Fish Among Anglers
}

By Tiffany Jonick, Elizabeth L. Anderson, Sharon Lin, Coral M. Bruni, P. Wesley Schultz, Stephen Groner, and Frankie Orrala

\begin{abstract}
In Southern California, white croaker caught within proximity to the Palos Verdes Shelf Superfund Site have been found to be contaminated with such high levels of dichloro-diphenyl-trichloroethane and polychlorinated biphenyls that fish consumption advisories recommend that this fish should not be consumed. In response to this risk, the U.S. Environmental Protection Agency has initiated an Angler Outreach Program designed to reduce the quantity of contaminated fish (specifically, white croaker) anglers bring back to the community. The current project describes the development of a community-based social marketing campaign targeting anglers, and reports results comparing the number of white croaker anglers took back to the community before and after a strategic intervention. Results indicate that the program was highly successful in positively modifying angler behavior. Postintervention results demonstrate that the strategic intervention reduced the number of white croaker entering the community by $93 \%$, and also produced a large reduction in the percentage of anglers who left the fishing site with white croaker. In addition, anglers reported an increase in positive behavior with regard to their white croaker consumption habits.
\end{abstract}




\section{Introduction}

Public outreach programs are notoriously difficult to evaluate and, more often than not, ineffective at changing behavior. In recognizing the limitations of generic public outreach programs, the Fish Contamination Education Collaborative (FCEC) drew heavily on the well-established principles of social marketing. Utilized for over 30 years both domestically and abroad, social marketing programs have been applied across a range of domains, but particularly to the improvement of public health conditions (De Gruchy \& Copel, 2008; Harvey, 2000; Lefebvre \& Flora, 1988; Reger, Wootan, Booth-Butterfield, \& Smith 1998; Rothschild, 2000; Walsh, Rudd, Moeykens, \& Moloney 1993) and more recently, fostering sustainable environmental behaviors (Andreasen, 1995).

In the current program, we adopted the community-based social marketing (CBSM) strategy proposed by McKenzie-Mohr (2000, 2002; McKenzie-Mohr \& Smith, 1999; Oskamp \& Schultz, 2006; Schultz \& Tabanico, 2008). The CBSM approach provides a specific set of steps emphasizing person-to-person channels of communication. The CBSM approach focuses on formative research (which includes identifying a target audience, target behaviors, and barriers and benefits associated with the target behavior), pilot testing, an intervention, and evaluation.

While the approach has proven particularly effective in promoting conservation behaviors, there has been scant research applying the strategy to reduce both public health and environmental risks faced by consumers. Additionally, the current work differs from previous public health campaigns utilizing a social marketing framework in that it encourages a target population to make a single behavior change to reduce risk, as opposed to improving the overall health of a population by modifying a number of behaviors.

Social marketing provides a mechanism for solving social problems through the application and reinterpretation of traditional marketing concepts (Andreasen, 1995; Neiger et al., 2001; Walsh et al., 1993). Unfortunately, many social marketing programs overemphasize passive forms of communication and information-rich messages. In contrast to such information-driven campaigns, CBSM aims to promote changes in behavior within a target population by focusing outreach efforts on specific behaviors and working at the community level through direct contact with individuals (McKenzie-Mohr \& Smith, 1999; Schultz, 2002; Schultz \& Tabanico, 2008). CBSM is used to create an effective message before implementing the larger social marketing intervention across a community by utilizing audience analysis within a target population for the purpose of message distribution (Alcalay \& Bell, 2001; Neiger et al., 2001; Walsh et al., 1993). As a result, CBSM principles are 
especially well-adapted for translating complex scientific messages and behavior change strategies into effective out- reach programs and communication campaigns (Lefebvre \& Flora, 1988, p. 300).

\section{Social problem}

The Palos Verdes Shelf (PVS) Superfund Site is located within the Southern California Bight, an area of the coastal Pacific Ocean between Point Conception and San Diego, CA. The site consists of contaminated sediments spanning a 17 square mile area about 1 to 3 miles offshore from the Palos Verdes peninsula. For nearly 40 years, the pesticides dichloro-diphenyl-trichloroethane (DDT) and polychlorinated biphenyls (PCBs) were discharged from several industrial sources into the sewers and were released into the ocean waters of the PVS. The DDT and PCBs mixed with the suspended solids in the discharge flowing out of the sewer outfalls and settled to the ocean floor to form a large sediment deposit. As a result, 110 tons of DDT and 10 tons of PCBs now cover the large expanse of ocean floor between Point Vicente in the northwest and Point Fermin in the southeast.

The mobility of the site's contaminants allows for the biological uptake of both DDT and PCBs by organisms that inhabit the site; and hence, human beings who are linked via the food web to those organisms. As a result, the largest threat to human health is the consumption of affected fish within the red zone, or the areas with the highest concentration of DDT and PCBs. Due to this area's high contaminant concentration, fish caught in the red zone are at the highest risk for contamination exposure, particularly white croaker. White croaker caught within the PVS site contain higher levels of DDT and PCB compared to other locally caught fish due to white croaker's bottom-dweller feeding habits, small home range, and ineffective digestion of the contaminants. Moreover, white croaker is the third most commonly caught fish in Los Angeles County with a high consumption rate relative to catch rate (Allen, Velez, Diehl, McFadden, \& Kelsh 1996).

The risks associated with the consumption of DDT- and PCB-contaminated fish like the white croaker include reproductive impairment, neurological damage, an increased risk of cancer, noncancerous chronic health problems, and liverdam- age. Immediate exposure to these contaminants in fish does not produce instant health effects; however, prolonged exposure to these contaminants leads to build up within the body, and consequently, increased health risks. The potential health effects associated with the consumption of contaminated white croakerdepend on a number of factors, including the chemical concentration within the fish, consumption rate, frequency of consumption, fish preparation methods, as well as individual 
consumer characteristics such as age, gender, life style, and health history.

\section{Methods}

The following study represents the efforts of the Angler Outreach Program, an arm of the U.S. Environmental Protection Agency's (USEPA) FCEC, which was established in response to the human health risks posed by the contaminated sediments along the PVS site. The primary objective of the Angler Outreach program is to design an effective communication strategy aimed at reducing the consumption of potentially contaminated locally caught white croaker among pier and shoreline anglers in Los Angeles and Orange counties. A central compo- nent of this communication strategy was the development and implementation of the Take Home Fish Assessment (THFA), which was based on CBSM tactics and techniques. In rolling out the THFA, FCEC first conducted formative research to identify the target audience as well as the barriers and benefits associated with the targeted behavior change through the use of a preintervention survey. The preintervention survey also served the vital purpose of determining preintervention figures related to angler behavior with regard to white croaker. The information garnered from the preintervention survey was then used to develop the social marketing intervention. As opposed to engaging in a traditional information-driven campaign, the Angler Outreach Program focused outreach efforts on promoting a singular behavior change message (the release of white croaker) through direct contact with individual anglers. Intervention materials were pilot-tested within a sample population to ensure clarity and effectiveness and were then rolled-out on a larger scale. Following the intervention, a postintervention survey was conducted to evaluate the effectiveness of the THFA.

\section{Formative research}

\section{Audience analysis}

FCEC identified that within the angling community, those leaving piers with white croaker in the red zone are at the highest risk for contamination exposure. This target subgroup was further refined by restricting angling locations to two fishing locations: Belmont Pier and Rainbow Harbor (Figure 1). Belmont Pier and Rainbow Harbor were selected after a thorough review of nine fishing locations: Belmont, Pier J, Rainbow Harbor, Marina Stadium, Long Beach Marina, Cabrillo Pier, Cabrillo Breakwall, White Point, and Cherry/Junipero Beach. These nine piers were evaluated based on angler traffic, the presence of signs warning against the consumption of white croaker, the quantity of white croaker caught, and red zone proximity. To control for 
seasonal availability in white croaker populations as well as in angler behavior, FCEC surveyed a nonintervention site (Belmont Pier) in addition to the intervention site (Rainbow Harbor). Prior to this evaluation, as part of the larger Angler Outreach Program, Belmont Harbor had received regular outreach by Heal the Bay - one of FCEC's partnering organizations that help administer the Angler Outreach Program - concerning the risks associated with the consumption of white croaker. Rainbow Harbor, however, had not received outreach prior to this assessment. Participants consisted of 234 anglers from the two target piers: Belmont Pier and Rainbow Harbor. The majority of anglers surveyed were Latino (52\%), followed by African American (15\%), White European=American (11\%), Filipino (9\%), and other (13\%). Other ethnicities included Cambodian (2\%), Chinese (1\%), Native American/Alaskan ( $<1 \%$ ), Vietnamese (3\%), and Pacific Islander (2\%). In terms of gender, $90 \%$ of anglers were male $(n=210), 7 \%$ of anglers were female $(n=18)$, and $3 \%$ of anglers were unknown $(n=6)$. Fully $68 \%$ of the surveys were conducted in English, with the remaining surveys conducted in Spanish.

\section{Target behaviors, barriers, and motivators}

A preintervention survey was used to identify the motivators and barriers acting upon the desired behavior change; in this case, the release of white croaker back into the ocean. In the preintervention survey, anglers were asked about the factors that would motivate them to release a contaminated fish, like the white croaker, back in the ocean. Anglers were also surveyed on the barriers restricting them from engaging in the desired behavior change. From this survey, the following motivators and barriers were identified. Approximately $73 \%$ of anglers reported that a health official directing them to release white croaker would be a very strong motivator for engaging in the behavior change. In addition, approximately $70 \%$ of anglers reported that protecting the health of their children would also be a very strong motivator. With regard to barriers, approximately 53\% of anglers said that not knowing white croaker is contaminated was the strongest barrier preventing them from releasing white croaker. 


\section{Map of Study Location}

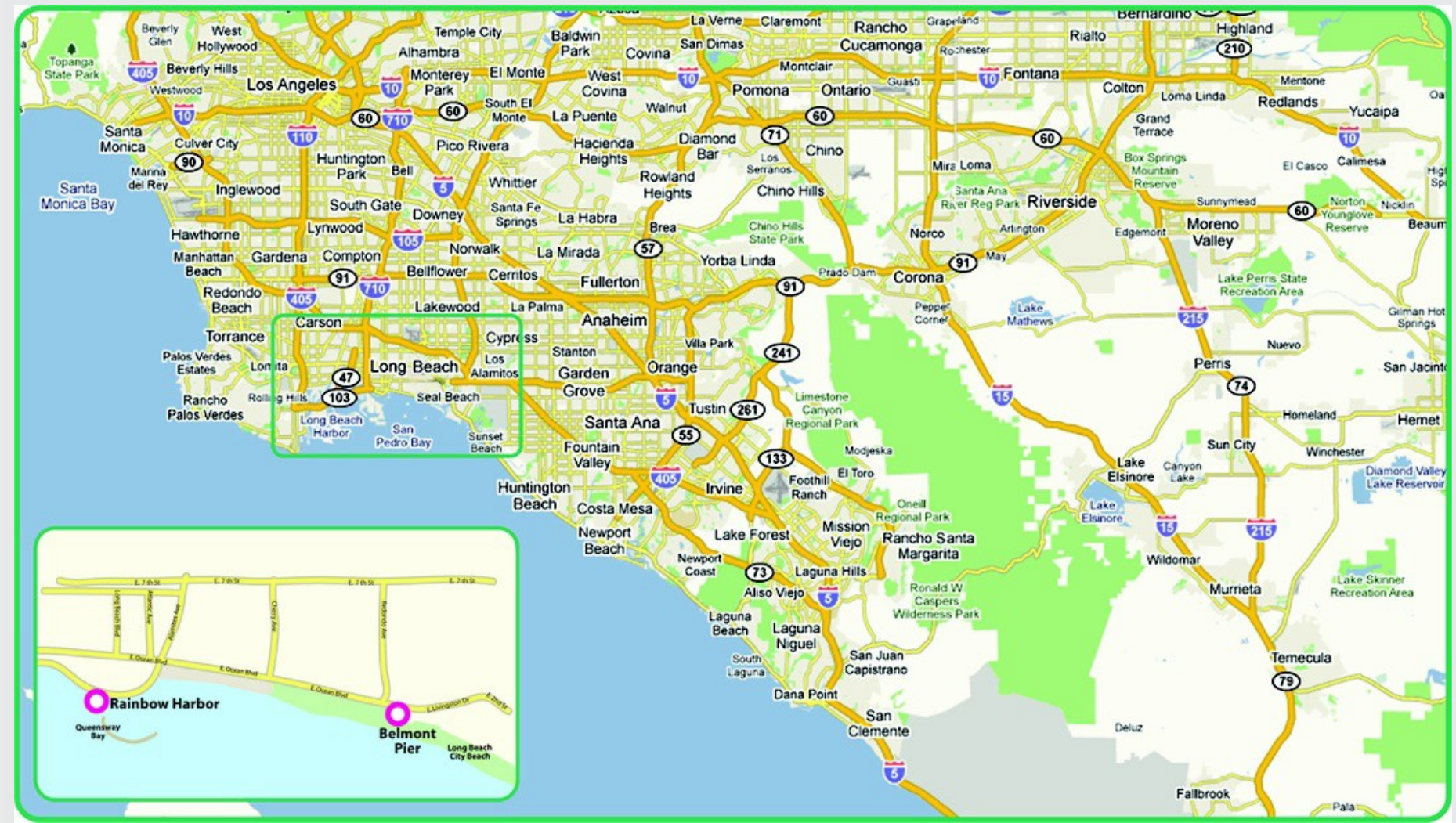



These motivators and barriers were integrated into the messaging strategy, based largely on the development and distribution of a Tip Card that encouraged anglers to release white croaker back to the ocean when caught from the red zone (Figure 2). Capturing the motivators identified in the preintervention period, one side of the card illustrates a young child being treated by a physician. The image of the child reinforces the urgency to protect the health of the angler's family while the image of the physician reinforces the legitimacy of the message. To overcome the barrier associated with a lack of contamination knowledge, the card also contains a large, clearly visible image of a white croaker along with corresponding text encouraging the release of this potentially contaminated fish.

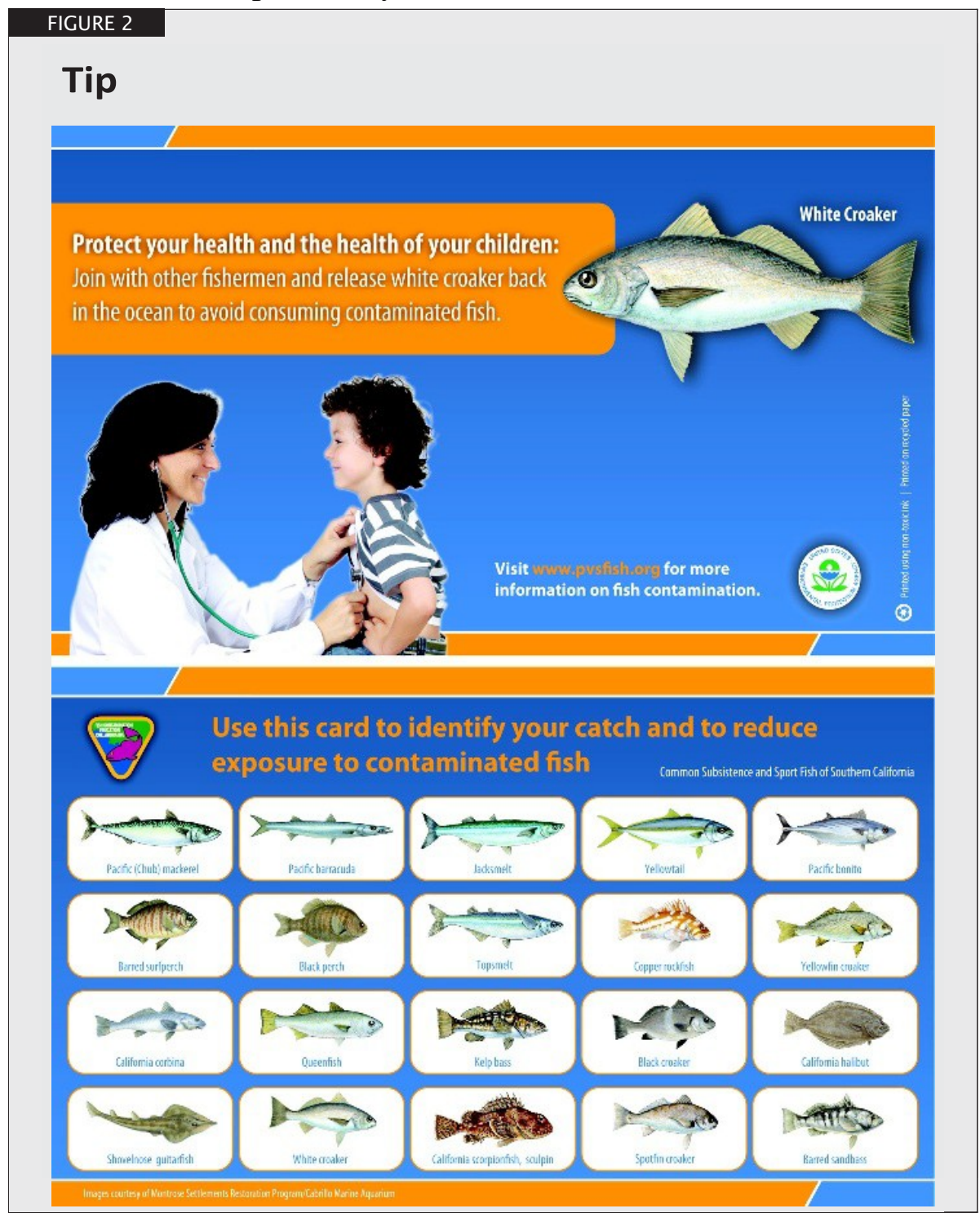




\section{Pilot-testing}

After developing the Tip Card, FCEC pilot-tested the material to gauge how effectively the Tip Card would be received by the target audience. During the pilottesting period, FCEC outreach workers conducted a series of five informal interviews with anglers at the intervention site. During the informal interviews, outreach workers asked a series of questions relating to the material's comprehensibility, usability, and aesthetic appeal. After receiving favorable responses during the informal interviews, FCEC moved forward with the intervention period.

\section{Intervention}

Following the pilot test, an intervention was conducted from September to October 2008 ( $N$ 300) utilizing the Tip Card coupled with interactions with outreach workers. In distributing the Tip Card, FCEC outreach workers emphasized the program's recommended behavior: release white croaker caught in the red zone. In distributing the Tip Card, FCEC outreach workers provided context to the issue by explaining why and how white croaker became contaminated and also emphasized the negative health effects associated with consumption of this potentially contaminated fish.

\section{Evaluation}

\section{Procedure}

After the intervention period, a postintervention survey was conducted to deter- mine if the intervention was effective in reducing the number of white croaker anglers brought back to the community. This survey was similar to the preintervention survey with several changes. In both the pre- and postintervention survey, actual behaviors were examined by recording the number of fish caught overall, the number of white croaker caught, and the techniques the anglers used in dealing with white croaker. In recording the number of fish and white croaker caught, outreach workers identified and counted the fish in each angler's fishing bucket; they recorded angler behavior toward white croaker by posing a series of targeted selfreported questions.

In both sets of surveys, anglers were also asked to identify their ethnicity, and the interviewers recorded the language in which the survey was conducted as well as the gender of the angler. Awareness of white croaker contamination was measured by asking anglers to correctly identify white croaker when presented with a card depicting several fish species. Outreach workers also measured awareness by asking 
anglers if they had come into contact with information, written or verbal, regarding white croaker contamination. The postintervention surveyed anglers regarding their exposure to the Tip Card used during the intervention period to distinguish anglers who had previously received outreach from those who had not for evaluation purposes.

Data from the preintervention survey was collected in Belmont Pier (nonintervention site) and Rainbow Harbor (intervention site) from May to June 2008 $(N=177)$. Postintervention data were collected from both piers from October to November 2008 ( $N^{1 / 457)}$. Both pre- and postintervention surveys were collected at Belmont Pier over the span of seven weekend days and at Rainbow Harbor over the course of nine weekend days. For both the pre- and postintervention surveys, outreach workers were not in the presence of participants while they were fishing to eliminate observation bias. While collecting data, outreach workers were stationed at the end of the pier and only approached individuals leaving the pier who were carrying fishing tack. If the outreach worker encountered a group of anglers, he or she would randomly approach one potential participant from the group. The outreach worker then screened the angler by asking the participant if he or she had been fishing. If the angler answered yes, the outreach worker would ask the participant if he or she would be willing to participate in a brief survey. If the participant agreed, the outreach worker proceeded to administer the survey.

For both pre- and postintervention surveys, outreach workers verbally posed each question to the respondent. For both surveys, the outreach worker also asked permission to count the number of fish that the angler possessed. If granted permission, the outreach worker counted and recorded the number of white croaker and other fish that the angler possessed in his or her bucket. After completing the survey, the outreach worker thanked the angler for his or her participation.

\section{Risk assessment}

The aim of the THFA was to reduce the aforementioned health risks resulting from prolonged exposure to contaminated fish. In determining the campaign's effect on risk reduction, FCEC collected and analyzed ten white croaker from the intervention site and nine white croaker from the nonintervention site for DDT and PCB concentrations. Using these analyses, the chemical intake associated with the ingestion of carcinogenic and noncarcinogenic constituents in fish were calculated to understand how the THFA might reduce the risk associated with the consumption of white croaker (USEPA 1989, 2000). Cancer slope factors developed by the USEPA represent upper-bound estimates; therefore, any cancer risks generated in this 
assessment should be regarded as an upper bound on the potential cancer risks rather than representations of true cancer risk. The actual cancer risk is likely to be less than that predicted (USEPA, 1989).

In addition, although synergistic or antagonistic interactions might occur between cancer-causing chemicals and other chemicals, information is generally lacking in the toxicological literature to predict quantitatively the effects of these potential interactions. Therefore, cancer risks will be treated as additive within an exposure route in this assessment. This is consistent with the USEPA guidelines on chemical mixtures (USEPA, 2000).

\section{Results}

Our analyses examined the impact of the THFA's intervention, designed to reduce the amount of white croaker leaving the piers and entering communities. The evaluation design was a 2 (pier: intervention site, nonintervention site) x 2 (time: preintervention, postintervention) quasiexperiment. Data from the pre- intervention survey were collected from Belmont Pier (nonintervention site) and Rainbow Harbor (intervention site) from May to June $2008(N=177)$. Post- intervention data were collected from October to November $2008(N=57)$. Three surveys from the initial data collection were removed from the analyses due to incomplete data.

Our first analysis focused on the number of white croaker leaving the piers, using data obtained by counting the number of white croaker in each angler's bucket. These scores were analyzed using a factorial ANOVA, with the time of data collection (2: pre- or postintervention) and the location of the pier (2: intervention or nonintervention site) as independent variables. There was a significant main effect of time, $F(1,227)=4.12, p<.05$, such that there were significantly more white croaker leaving the piers preintervention ( $M=.48$ white croaker per angler) than postintervention ( $M=.13$ white croaker per angler). There was no main effect of location. However, there was a significant interaction between time of data collection and location, $F(1,227)=5.23, p<.05$. Relatively no change occurred at the nonintervention site from pre $(M=.16)$ to post $(M=.20)$; however, there was a dramatic decrease in the number of white croaker leaving the intervention site from preintervention $(M=.81)$ to postintervention $(M=.06)$. This represents a $93 \%$ reduction in the number of white croaker leaving the intervention pier.

While there were statistical differences in the amount of white croaker leaving the pier in the intervention group, this reduction could be due to other factors that occurred at the piers, such as the total number of fish being caught. To account for the differences, a proportion score was created by taking the totalnumber of white 
croaker per angler leaving the pier and dividing by the total number of fish (including white croaker) per angler leaving the pier. Thus, an angler leaving the pier with only one fish (white croaker) would receive a proportion score of 1 ; whereas an angler leaving the pier with 10 fish (one of which was white croaker) would receive a score of .1. The proportion scores were analyzed using a factorial ANOVA, with the time of data collection (2: pre- or postintervention) and the location of the pier (2: intervention or nonintervention site) as independent variables. There was not a main effect of time of data collection or location. However, there was a significant interaction between time of data collection and location, $F(1,227)=10.01, p<.01$. As seen in Figure 3, a small increase occurred at the nonintervention site from pre ( $M=.03)$ to post $(M=.12)$, with angers leaving the pier with a higher proportion of white croaker post than pre. However, there was a significant decrease in the proportion of white croaker leaving the intervention site from preintervention ( $M=$ $.22)$ to postintervention $(M=.05)$. After outreach, the percent of each angler's catch that was white croaker decreased, whereas the nonintervention site exhibited an increase in the percentage of white croaker leaving the pier.

Another goal of the assessment was to determine the number of anglers leaving the piers with white croaker in relation to those anglers leaving with other species of fish or no fish. A proportion score was created by taking the number of anglers leaving the pier with white croaker and dividing by the total number of anglers leaving the pier. By comparing this proportion score against pre- and postintervention figures, this information provides the basis for determining whether or not the intervention was successful. Additionally, this information also helps to establish whether small concentrations of anglers are leaving with large amounts of white croaker or if this distribution is more dispersed.

During the preintervention period, 9\% of anglers at the nonintervention site left with white croaker, while $30 \%$ of anglers at the intervention site left with white croaker. The low proportion of anglers leaving the nonintervention site with white croaker compared to the relatively high proportion observed at the intervention site could be attributed to previous angler outreach efforts at the nonintervention site. During the postintervention period, $12 \%$ of anglers at the nonintervention site left with white croaker, while only $6 \%$ of anglers at the intervention site left with white croaker. 
Interaction Between Time of Data Collection (pre or post) and Location (Nonintervention or Intervention Site) in Proportion of White Croaker Leaving the Pier

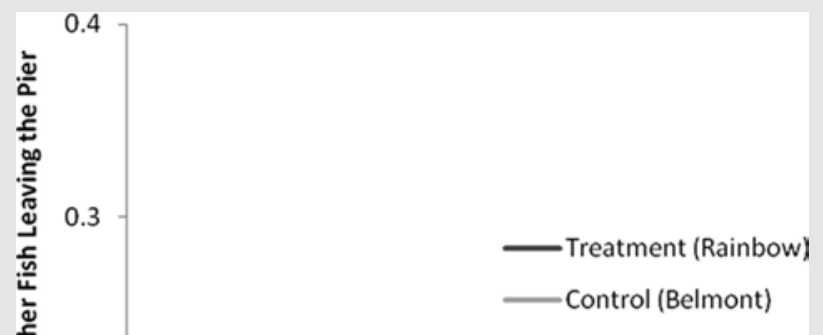

0

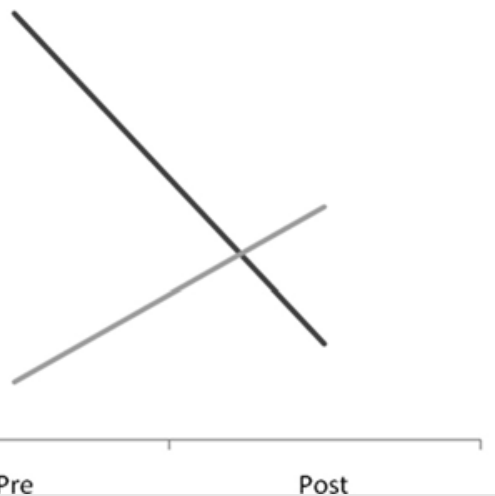


To assess the changes in the habits and intentions with regard to the fish, anglers were asked what they do with the white croaker they catch (Table 1). After outreach, anglers at both piers demonstrated an increase in positive forms of behavior (release or use as bait). The number of anglers who reported releasing the white croaker showed the largest improvement (23\% increase) in the intervention site over preintervention figures in this category. The number of anglers who reported giving the fish to their friends and family showed the secondlargest improvement, with a decrease of $15 \%$ at the nonintervention site and a decrease of $36 \%$ at the intervention site. Finally, the number of anglers who reported eating the white croaker showed a marked improvement at the intervention site, boasting a 22\% decrease while anglers reporting this behavior increased by $3 \%$ at the nonintervention site. A 2 × 2 chi square test showed no difference in behaviors seen in the nonintervention group, $X^{2}(1)$ $=.46, p=$ ns. However, there was a difference in behaviors seen in the intervention group, $X^{2}(1) \frac{1}{4} 11.90, p<.01$, such that after outreach the incorrect behaviors were significantly lower (Figure 4 ).

Table 1

\section{Survey Question: What Do You Usually DO With the White Croaker You Catch?}

\begin{tabular}{|l|l|l|l|l|}
\hline & $\begin{array}{l}\text { NONINTERVENT } \\
\text { ION SITE (PRE) }\end{array}$ & $\begin{array}{l}\text { INTERVENTION } \\
\text { SITE (PRE) }\end{array}$ & $\begin{array}{l}\text { NONINTERVENT } \\
\text { ION SITE (Post) }\end{array}$ & $\begin{array}{l}\text { INTERVENTION } \\
\text { SITE (POST) }\end{array}$ \\
\hline Eat & 15 & 12 & 1 & 4 \\
\hline $\begin{array}{l}\text { Give to } \\
\text { friends/family }\end{array}$ & 15 & 10 & 0 & 1 \\
\hline $\begin{array}{l}\text { Catch and } \\
\text { release }\end{array}$ & 51 & 54 & 28 & 18 \\
\hline Use for bait & 0 & 3 & 3 & 2 \\
\hline
\end{tabular}




\section{Risk reduction}

For the purpose of the USEPA Superfund risk assessment, USEPA makes conservative assumptions in calculating the excess cancer risks associated with consuming contaminated white croaker to design a remedy that is protective of the most sensitive population (USEPA, 1989, 2000). In this calculation specifically, the average consumer cancer risk was calculated using the following assumptions:

- An ingestion rate of $21.4 \mathrm{~g} /$ day (site specific for average end fish consumers, based on the Santa Monica Bay Seafood Consumption Study conducted by the Santa Monica Bay Restoration Project [Santa Monica Bay Restoration Project 1994]).

- The individual is eating the fish 365 days/year for 13.8 years.

- The individual's body weight is $70 \mathrm{~kg}$.

- The individual is only eating fish fillet not the whole fish.

\section{FIGURE 4}

\section{Correct and Incorrect Handling Techniques for White Croaker by Site}

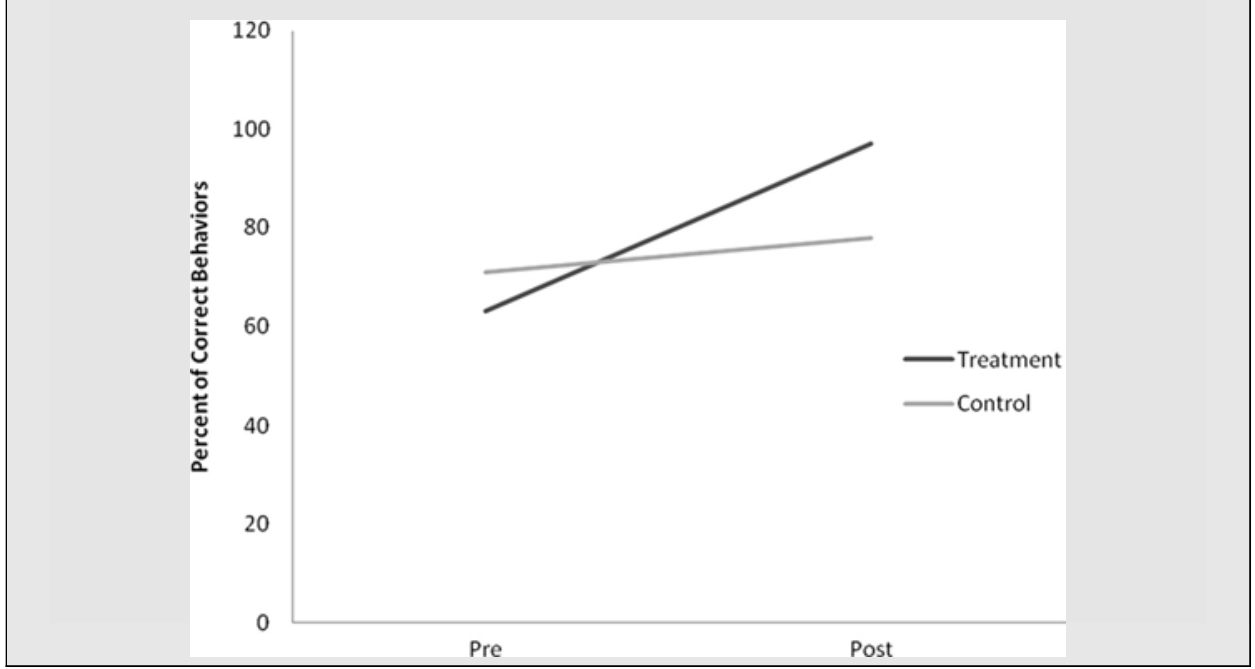


Ten white croaker from the intervention site and nine white croaker from the nonintervention site were collected and analyzed for DDT and PCBs. The excess cancerrisks associated with the average consumers are $2.4 \times 10^{-5}$ (2.4 excess incidences of cancer in 100,000 people) eating the white croaker fillet caught at the intervention site and $1.8 \times 10^{-5}$ (1.8 excess incidences of cancer in 100,000 people) eating the white croaker fillet caught at the nonintervention site, respectively. For all analyses, we will use $2.1 \times 10^{-5}$ (2.1 excess incidences of cancer in 100,000 people), which is the average excess cancer riskbetween the two sites.

To calculate the risk reduction associated with the THFA program, the total population of marine anglers in Los Angeles and Orange counties needs to be estimated. To do this, the total population in Los Angeles and Orange counties (13,450,060; U.S. Census Bureau, 2008a, 2008b) is compared to the total population of California (36,756,666; U.S. Census Bureau, 2008c) to create a percent- age (36.59\%). This percentage is then used to calculate an estimated number of marine anglers in Los Angeles and Orange counties by taking 36.59\% of California's total marine angling population (1,100,000; U.S. Department of Commerce, 2007), which results in an estimated total marine angling population in Los Angeles and Orange counties of 402,490. Using the estimated cancer risk of $2.1 \times 10^{-5}$ of the estimated population of 402,490 marine anglers and assuming they are all average consumers, there would be about 8.45 potential excess incidences of cancer due to ingesting DDT and PCBs in white croaker fillets over the projected 13.8-year period.

Eliminating the practice of leaving piers with white croaker is a $100 \%$ effective means of reducing the excess cancer risk associated with the consumption of this fish and can be used to calculate the effectiveness of using the THFA to reduce risk. In our study, there was a 93\% decrease obtained at the intervention site in the amount of white croaker leaving the piers and entering communities. Thus, a 93\% reduction of risk would bring the excess incidences of cancer due to ingesting DDT and PCBs in white croaker fillets down to $1.47 \times 10^{-6}$, or approximately .59 potential incidences among the 402,490 anglers in the affect region. Therefore, if the THFA program reached the entire marine angling population in Los Angeles and Orange Counties (402,490 anglers), eight people could potentially be saved from developing cancer. 


\section{Discussion}

Results of the THFA suggest that CBSM is an effective tool for public health campaigns, and specifically for programs designed to reduce the risks associated with the consumption of contaminated fish. For instance, angler behavior with regard to white croaker consumption and habits improved dramatically from pre to post at the intervention site, yet showed no improvement in the nonintervention site. Moreover, the number of white croaker removed decreased by $93 \%$ from pre to post at the intervention site. Compounding this effect, the nonintervention site still exhibited a small increase in the number of white croaker removed, even though fewer white croaker were present at both locations after the intervention. Finally, the proportion of anglers leaving with white croaker reduced by $24 \%$ from pre to post at the intervention site: during the pretest, $30 \%$ of the anglers leaving the pier had at least one white croaker, whereas only $6 \%$ of anglers had white croaker during the postintervention period. By comparison, the proportion of anglers leaving with white croaker at the nonintervention site increased by 3\%: from $9 \%$ at pre to $12 \%$ at post.

In addition to achieving reductions in the removal of white croaker, another goal of this study was to understand the reduction of excess cancer risks associated with the consumption of white croaker through the efforts of the THFA. After the program, the excess cancer risk associated with the consumption of contaminated white croaker was reduced by $93 \%$. We estimate that over a 13.8-year period, our outreach program would prevent approximately eight individuals from developing cancer. This suggests that the THFA is successful in reducing the potential excess cancer risk associated with the consumption of white croaker.

\section{Implications for Social Marketing}

It is useful to speculate about the causal relationship between the survey results and the intervention. The program used three unique elements during outreach to promote behavior change that are particularly noteworthy, including the intervention material's focus on a simple, singular message; the use of direct one-on- one outreach; and the utilization of strong visual images to convey the overall message of the THFA. These unique methods carry an implication for social marketing practitioners in a range of disciplines.

As opposed to most public health campaigns, this intervention focused on modifying a single consumption pattern as opposed to an overall dietary behavior change. Consumer research has demonstrated that community members feel confused 
by the number of public health messages to which they are exposed (American Dietetic Association, 1995). For this reason, the THFA's intervention material focused on a single, straightforward message: release white croaker. As other studies have tested and hypothesized, community members are typically more willing to take on one behavior change at a time as opposed to undertaking a comprehensive life-style change(Regger etal., 1998). Research has also shown that messages which are clearly articulated are more likely to be comprehended and followed than those that are more complex (Brunetti, Tomasik, \& Taraba, 2000; Regger et al., 1998).

The intervention may have also been successful as a result of how the message was portrayed. Utilizing an image of a health professional was meant to charge the message with a sense of authority and legitimacy, while the child image was meant to create awareness that consuming white croaker could affect the health of the angler's child and/or family. Communications campaigns have demonstrated that utilizing tangible visual images can be extremely influential in not only creating awareness, but also changing behavior (Horn, 1999; Roam, 2008).

Moreover, the intervention material images were specifically tailored to the target population based on information collected during the formative research period. The preintervention survey indicated that the majority of anglers would be more motivated to release white croaker if instructed to do so by a health official or if the health of their children would be compromised if the prescribed behavior was not followed. Adding a "personal touch" to the outreach materials by tailoring them to the target population could have encouraged positive behavior changes by increasing the impact of the message (Schultz \& Tabanico, 2008). In addition to personalizing outreach materials, FCEC staff also conducted direct outreach to pier anglers during the intervention to educate them on the risks of consuming potentially contaminated white croaker. Numerous studies have demonstrated that personal contact is the most powerful outreach mechanism in influencing individual attitudes and behaviors (McKenzie-Mohr \& Smith, 1999; Neiger et al., 2001; Schultz, 2002; Schultz \& Tabanico, 2008).

\section{Conclusion}

Utilizing the unique methods of CBSM carries an implication for social marketing practitioners in a range of disciplines. In this study, FCEC was successful in using CBSM and outreach to reduce the risk associated with contaminated fish by positively modifying white croaker consumption behaviors. As in this study, CBSM can be used to modify behavior, reduce risk, and produce the desired outcomes in many social marketing campaigns. 


\section{About the Authors}

Tiffany Jonick, M.A., is a project manager with S. Groner Associates Inc., overseeing and implementing the community outreach and education efforts for FCEC on behalf of USEPA. She has spoken and written about social marketing, presenting at the Headwaters to Ocean conference in Southern California and she is published in Water and Wastewater News. Tiffany holds a master of arts in journalism from the University of Southern California in addition to a bachelors of arts in history and sociology from the University of Notre Dame.

Elizabeth L. Anderson, M.S., is a practitioner of community-based social marketing with S. Groner Associates Inc. She coordinates the multiethnic outreach component of FCEC, facilitating the involvement of over 20 community-based organizations (CBOs) and governmental agencies, while also executing research and evaluation efforts related to the program. Elizabeth is fluent in English and Spanish and has extensive experience working with underserved Asian and Hispanic community members in the Southern Californian basin through her work with FCEC. She received a master of science in nature, society and environmental policy from the University of Oxford as well as a bachelor of science in policy and management and a bachelor of arts in Hispanic studies from Carnegie Mellon University.

Sharon Lin, M.S., is a project manager with the U.S. Environmental Protection Agency Superfund program office. Sharon is responsible for managing clean-up activities at two contaminated sediment Superfund sites in California. She has 15 years of experience working in the field of contaminated sediment management, ranging from technical policy to community-related issues. Sharon holds a bachelor of science and a master of science in civil engineering (with an emphasis in environmental engineering) from San Jose State University. She is a registered professional engineer in California.

Coral M. Bruni, M.A., is a project manager at Action Research, Inc. Her research interests are in applied social psychology, with an emphasis in applied social cognition, conservation psychology, and statistics. Coral has completed numerous studies of environmental attitudes and implicit social cognition. Her most recent work has focused on the development of environmental attitudes in children. She has worked on projects for a variety of organizations, including the U.S.

Environmental Protection Agency, Keep America Beautiful, and the San Diego Association of Governments. 
P. Wesley Schultz, Ph.D., is professor of psychology at California State University, and senior consulting scientist at Action Research Inc. His research interests are in applied social psychology, social influence, and statistics. Recent books include Social Psychology: An Applied Perspective (2000, Prentice-Hall), Psychology of Sustain- able Development (2002, Kluwer), and Attitudes and Opinions (2005, Lawrence Erlbaum). His current work focuses on social norms and the importance of social norms in community-based social marketing programs. He has worked on projects for a variety of organizations, including the National Institute of Justice, U.S. Environmental Protection Agency, National Institutes of Health, and the California Integrated Waste Management Board. Website: www.csusm.edu/schultz.

Stephen Groner is the founder and president of S. Groner Associates Inc., a social marketing and community relations firm in Southern California. Stephen has been working with community-based social marketing for nearly a decade. His efforts have been highlighted by the California Integrated Waste Management Board as model programs for local government. Stephen has directed the implementation of all major SGA campaigns, including the firm's current work with FCEC. He holds a bachelor of science in civil and environmental engineering from the Uni- versity of Wisconsin and is a California registered civil engineer.

Frankie Orrala is responsible for the day-to-day implementation of FCEC's Angler Outreach Program at Heal the Bay. Frankie has developed nontraditional partnerships within the local Spanish-speaking community, providing a direct link to the region's underserved Hispanic population. Prior to joining Heal the Bay, Frankie worked as a fishing observer on board a Japanese-Ecuadorian Fleet for the Ecuadorian National Fishing Institute in the Galapagos Islands. Frankie holds a bachelor of science from the University of Guayaquil in marine biology.

\section{Acknowledgments}

This study was made possible through the leadership, guidance, and financial support provided by the USEPA Superfund Program, Region IX. First, we give our special thanks to Dr. Sophia Serda of USEPA Region IX for her invaluable contributions to the risk assessment portion of this manuscript. Second, we thank Heal the Bay and S. Groner Associates for conducting the study's field research as well as Action Research Team for their evaluation support. We also thank the following colleagues for their insightful comments and revisions on earlier ver- sions of this assessment: Robert Brodberg from the Office of Environmental Health 
Hazards Assessment, Marilyn Underwood from the California Depart- ment of Health Services, and Alyce Ujihara from the Environmental Health Investigations Branch. Last, we thank the FCEC partners for their continued support throughout the study's administration.

\section{References}

Alcalay, R., \& Bell, A. (2001). Strategies and practices in communitybased campaigns promoting nutrition and physical activity. Social Marketing Quarterly, 7(4), 3-15.

Allen, M. J., Velez, P. V., Diehl, D. W., McFadden, S. E., \& Kelsh, M. (1996). Demographic variability in seafood consumption rates among recreational anglers of Santa Monica Bay, California in 1991-1992. Fishery Bulletin (U.S.), 94, 597-610.

American Dietetic Association. (1995). Nutrition trends survey. Chicago, IL: Author.

Andreasen, A. R. (1995). Marketing social change: Changing behavior to promote health, social devel- opment and the environment. San Francisco, CA: Jossey-Bass.

Brunetti, G., Tomasik, H. H., \& Taraba, L. (2000). Social marketing tools used to support the development of a community-based physical activity initiative. Social Marketing Quarterly, 6(3), 93-99.

De Gruchy, J., \& Copel, D. (2008). “Listening to reason”: A social marketing stop-smoking campaign in Nottingham. Social Marketing Quarterly, 14(1), 5-17.

Harvey, P. D. (2000). Let every child be wanted: How social marketing is revolutionizing contra- ceptive use around the world. Social Marketing Quarterly, 6(4), 115-118.

Horn, R. E. (1999). Visual Language: Global Communication for the 21st Century. Bainbridge Island, WA: MacroVU.

Lefebvre, R. C., \& Flora, J. A. (1988). Social marketing and public health intervention. Health Education Quarterly, 15, 299-315.

McKenzie-Mohr, D. (2000). Promoting sustainable behavior: An introduction to community- based social marketing. Journal of Social Issues, 56, 543-554. 
McKenzie-Mohr, D. (2002). The next revolution sustainability. In P. Schmuck \& P. W. Schultz (Eds.), Psychology of sustainable development (pp. 19-36). Dordrecht, The Netherlands: Kluwer Academic.

McKenzie-Mohr, D., \& Smith, W. (1999). Fostering sustainable behavior: An introduction to com- munity-based social marketing. Gabriola Island, BC, Canada: New Society.

Neiger, B. L., Thackeray, R., Merril, R. M., Miner, K. M., Larsen, L., \& Chalkey, C. M. (2001). The impact of social marketing on fruit and vegetable consumption and physical activity among public health employees at the Utah department of health. Social Marketing Quarterly, 2(1), 10-28.

Oskamp, S., \& Schultz, W. (2006). Using psychological science to achieve ecological sustainability. In S. Donaldson, D. Berger, \& K. Pezdek (Eds.), Applied psychology: New frontiers and rewarding careers (pp. 81-106). Mahwah, NJ: Lawrence Erlbaum.

Reger, B., Wootan, M. G., Booth-Butterfield, S., \& Smith, H. (1998). 1\% or less: A community- based nutrition campaign. Public Health Reports, 113, 410-419.

Roam, D. (2008). The back of the napkin: Solving social problems and selling ideas with pictures. New York, NY: Portfolio=Penguin Group (USA).

Rothschild, M. L. (2000). Carrots, sticks, and promises: A conceptual framework for the management of public health and social issue behaviors. Social Marketing Quarterly, 6(4), 87-114.

Santa Monica Bay Restoration Project. (1994, June). Santa Monica Bay Seafood Consumption Study [Final report]. Westminster, CA: Southern California Coastal Water Research Project; and, Costa Mesa, CA: MBC Applied Environmental Sciences. 
Schultz, P. W. (2002). Knowledge, education, and household recycling: Examining the knowledge- deficit model of behavior changes. In T. Dietz \& P. C. Stern (Eds.), New tools for environmental protection: Education, information, and voluntary measures (pp. 67-82). Washington, DC: National Academy of Sciences.

Schultz, P. W., \& Tabanico, J. J. (2008). Community-based social marketing ad behavior change. In A. Cabaniss (Ed.), Handbook on household hazardous waste (pp. 133-156). Lanham, MD: Rowan and Littlefield.

U.S. Census Bureau. (2008a, July). Estimated Population for Los Angles County, CA. Population Division. Available from Census (http://www.census.gov/popest/estimates.html)

U.S. Census Bureau. (2008b, July). Estimated Population for Orange County, CA. Population Division. Available from Census (http://www.census.gov/popest/estimates.html)

U.S. Census Bureau. (2008c, July). Estimated Population for the State of California. Population Division. Available from Census (http://www.census.gov/popest/estimates.html)

U.S. Department of Commerce, National Oceanic and Atmospheric Administration, \& National Marine Fisheries Service. (2007). By the numbers: Saltwater fishing facts and figures for 2006. Washington, DC: Office of Sustainable Fisheries, Partnerships and Communications Division; Office of Science and Technology, Fisheries Statistics Division.

U.S. Environmental Protection Agency. (1989). Risk assessment guidance for superfund, volume I: Human health evaluation manual, Part A (EPA=540=1-89=002). Washington, DC: Author, Office of Solid Waste and Emergency Response, Toxics Integration Branch.

U.S. Environmental Protection Agency. (2000). Guidance for assessing chemical contaminant data for use in fish advisories, volume 2 (3rd ed., EPA 823-B-00-008). Washington, DC: Office of Water.

Walsh, D. C., Rudd, R. E., Moeykens, B. A., \& Moloney, T. W. (1993). Social marketing for public health. Health Affairs, 12, 104-119. 